\title{
Pulmonary hypertension: the importance of correctly diagnosing the cause
}

\author{
Sanjay Mehta ${ }^{1,2}$ and Jean-Luc Vachiéry ${ }^{3}$
}

\begin{abstract}
Affiliations: 'Southwest Ontario PH Clinic, London Health Sciences Centre, Victoria Hospital, Western University, London, ON, Canada. ${ }^{2}$ Pulmonary Hypertension Association of Canada, Vancouver, BC, Canada. ${ }^{3}$ Pulmonary Vascular Diseases Clinic, Dept of Cardiology, Cliniques Universitaires de Bruxelles - Hôpital Erasme, Brussels, Belgium.
\end{abstract}

Correspondence: Jean-Luc Vachiéry, Pulmonary Vascular Diseases Clinic, Dept of Cardiology, Cliniques Universitaires de Bruxelles - Hôpital Erasme, Brussels, Belgium. E-mail: JeanLuc.Vachieryderasme.ulb.ac.be

ABSTRACT Pulmonary hypertension $(\mathrm{PH})$ is a complex condition that can occur as a result of a wide range of disorders, including left heart disease, lung disease and chronic pulmonary thromboembolism. Contemporary $\mathrm{PH}$ patients are older and frequently have a multitude of comorbidities that may contribute to or simply coincide with their $\mathrm{PH}$. Identifying the cause of $\mathrm{PH}$ in these complicated patients can be challenging but is essential, given that the aetiology of the disease has a significant impact on the management options available. In this article, we present two cases that highlight the difficulties involved in obtaining a precise diagnosis of the cause of $\mathrm{PH}$ within the setting of multiple comorbidities. The importance of performing a comprehensive, multidimensional diagnostic work-up is demonstrated, in addition to the need to specifically consider cardiopulmonary haemodynamic data in the context of the wider clinical picture. The article also illustrates why achieving an accurate diagnosis is necessary for optimal patient management. This may involve treatment of comorbidities as a priority, which can ameliorate the severity of $\mathrm{PH}$, obviating the need to consider $\mathrm{PH}$-targeted medical treatment.

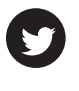

@ERSpublications

Multidimensional testing in $\mathrm{PH}$ is essential, especially when patients present with comorbidities http://ow.ly/kYJa305OIi2

\section{Introduction}

An accurate and timely diagnosis of pulmonary hypertension $(\mathrm{PH})$ and identification of the specific underlying cause of $\mathrm{PH}$ is essential to ensure that patients can be treated as early as possible with interventions that are appropriate for their diagnosis. Many effective PH-targeted therapies are available $[1,2]$ and when used in patients with appropriate types of $\mathrm{PH}$, predominantly those with World Health Organization (WHO) Group 1 pulmonary arterial hypertension (PAH), these therapies can lead to significant improvements in functional capacity, quality of life and outcome $[1,2]$. As the underlying cause and recommended management approach varies between patients, $\mathrm{PH}$-targeted therapies are not suitable for all $\mathrm{PH}$ patients [1, 2]. For example, PH-targeted pharmacotherapies have not been shown to be effective for WHO Group $2 \mathrm{PH}$ (PH due to left heart disease (LHD)) or WHO Group $3 \mathrm{PH}$ (PH due to lung disease and/or hypoxia) [3].

As the appropriate treatment differs between $\mathrm{PH}$ patients $[1,2]$, it is important to correctly identify the cause of $\mathrm{PH}$ in each patient. However, obtaining a definitive diagnosis can be difficult due to the increasing age of $\mathrm{PH}$ patient populations and the fact that these patients frequently present with significant comorbidities, such as obesity, systemic hypertension, diabetes and underlying respiratory disorders $[4,5]$.

Editorial comment in Eur Respir Rev 2016; 25: 361-363.

Received: Aug 192016 | Accepted after revision: Oct 182016

Conflict of interest: Disclosures can be found alongside this article at err.ersjournals.com

Provenance: The European Respiratory Review received sponsorship from Actelion Pharmaceuticals Ltd, Allschwil, Switzerland, for the publication of these peer-reviewed articles.

Copyright CERS 2016. ERR articles are open access and distributed under the terms of the Creative Commons Attribution Non-Commercial Licence 4.0. 
This article highlights the challenges associated with identifying the specific cause of $\mathrm{PH}$ in patients with significant comorbidities, and the importance of careful integration and interpretation of various multidimensional diagnostic tests in order to achieve this. Two patient cases are presented to illustrate the complexity of making a specific diagnosis of the cause of $\mathrm{PH}$ and the importance of achieving an accurate diagnosis in order to appropriately manage the disease.

\section{Case 1}

A 57-year-old male with newly identified $\mathrm{PH}$ was referred for assessment and treatment by the specialist $\mathrm{PH}$ clinic. He complained of a 1-year history of dyspnoea after walking up less than one flight of stairs, fatigue and ankle oedema, but no chest pain or orthopnoea. The patient had long-standing morbid obesity (body mass index (BMI) $\sim 55 \mathrm{~kg} \cdot \mathrm{m}^{-2}$ ), type 2 diabetes mellitus, systemic hypertension and obstructive sleep apnoea syndrome, and had previously been treated for atrial flutter. At the time of assessment, he was treated with two antihypertensive drugs, an antiarrhythmic drug and a diuretic. As shown in table 1, echocardiography indicated a high probability of $\mathrm{PH}$ and a normal left ventricular ejection fraction. Pulmonary function tests (PFTs) and blood gas analysis revealed no sign of lung disease. Pulmonary emboli were excluded by nuclear ventilation/perfusion $\left(V^{\prime} / Q^{\prime}\right)$ lung scanning. Functional capacity was maintained, as shown by the 6-min walking distance (table 1). $\mathrm{PH}$ was confirmed by right heart catheterisation (RHC) (table 1). Although the pulmonary artery wedge pressure (PAWP) was close to the cut-off point of $\leqslant 15 \mathrm{mmHg}$ commonly accepted to identify pre-capillary pulmonary hypertension (which includes PAH), as opposed to post-capillary pulmonary hypertension (which includes PH-LHD) $[1,2]$, it was considered unreliable due to the patient's obesity. Therefore, left heart catheterisation (LHC) was performed, which demonstrated an elevated left ventricular end-diastolic pressure (LVEDP) and an elevated diastolic pressure gradient $(\mathrm{DPG}=$ diastolic pulmonary arterial pressure (PAP)-LVEDP). This led to a final diagnosis of combined post- and pre-capillary pulmonary hypertension $(\mathrm{Cpc}-\mathrm{PH})$ (figure 1), in the context of heart failure with preserved ejection fraction ( $\mathrm{HFpEF}$ ) and obesity. The patient's obesity was subsequently treated successfully by bariatric surgery, which considerably improved his $\mathrm{PH}$.

\section{Case 2}

A 37-year-old female was referred to the specialist $\mathrm{PH}$ clinic with severe $\mathrm{PH}$ and right ventricular (RV) failure. She had recently been admitted to hospital with fatigue, dyspnoea and severe oedema, which had worsened over a period of 6 months. She was a former smoker of half a pack of cigarettes per day for

\begin{tabular}{|c|c|}
\hline Parameter & Assessment \\
\hline 6MWD m & 470 \\
\hline \multicolumn{2}{|l|}{ Echocardiography $\#$} \\
\hline $\mathrm{TRV} \mathrm{m} \cdot \mathrm{s}^{-1}$ & 4.51 \\
\hline RVSP mmHg & 91 \\
\hline TAPSE mm & $>18$ \\
\hline LVEF & Normal \\
\hline \multicolumn{2}{|l|}{ Right heart catheterisation } \\
\hline Systolic/diastolic PAP mmHg & $100 / 38$ \\
\hline Mean PAP mmHg & 61 \\
\hline PAWP mmHg & 16 \\
\hline $\mathrm{DPG} \mathrm{mmHg}$ & 22 \\
\hline $\mathrm{CO} \mathrm{L} \cdot \mathrm{min}^{-1}$ & 8.31 \\
\hline $\mathrm{Cl} \mathrm{L} \cdot \mathrm{min}^{-1} \cdot \mathrm{m}^{-2}$ & 3.2 \\
\hline PVR Wood units & 5.45 \\
\hline RAP mmHg & 14 \\
\hline $\mathrm{SvO}_{2} \%$ & 67 \\
\hline \multicolumn{2}{|l|}{ Left heart catheterisation } \\
\hline LVEDP mmHg & 19 \\
\hline $\mathrm{DPG} \mathrm{mmHg}$ & 23 \\
\hline
\end{tabular}

6MWD: 6-min walking distance; TRV: tricuspid regurgitation velocity; RVSP: right ventricular systolic pressure; TAPSE: tricuspid annular plane systolic excursion; LVEF: left ventricular ejection fraction; PAP: pulmonary arterial pressure; PAWP: pulmonary artery wedge pressure; DPG: diastolic pressure gradient; CO: cardiac output; $\mathrm{Cl}$ : cardiac index; PVR: pulmonary vascular resistance; RAP: right atrial pressure; $\mathrm{SvO}_{2}$ : mixed venous oxygen saturation; LVEDP: left ventricular end diastolic pressure. ${ }^{\#}$ : right ventricular size and right ventricular systolic function were not assessable due to poor imaging quality. 

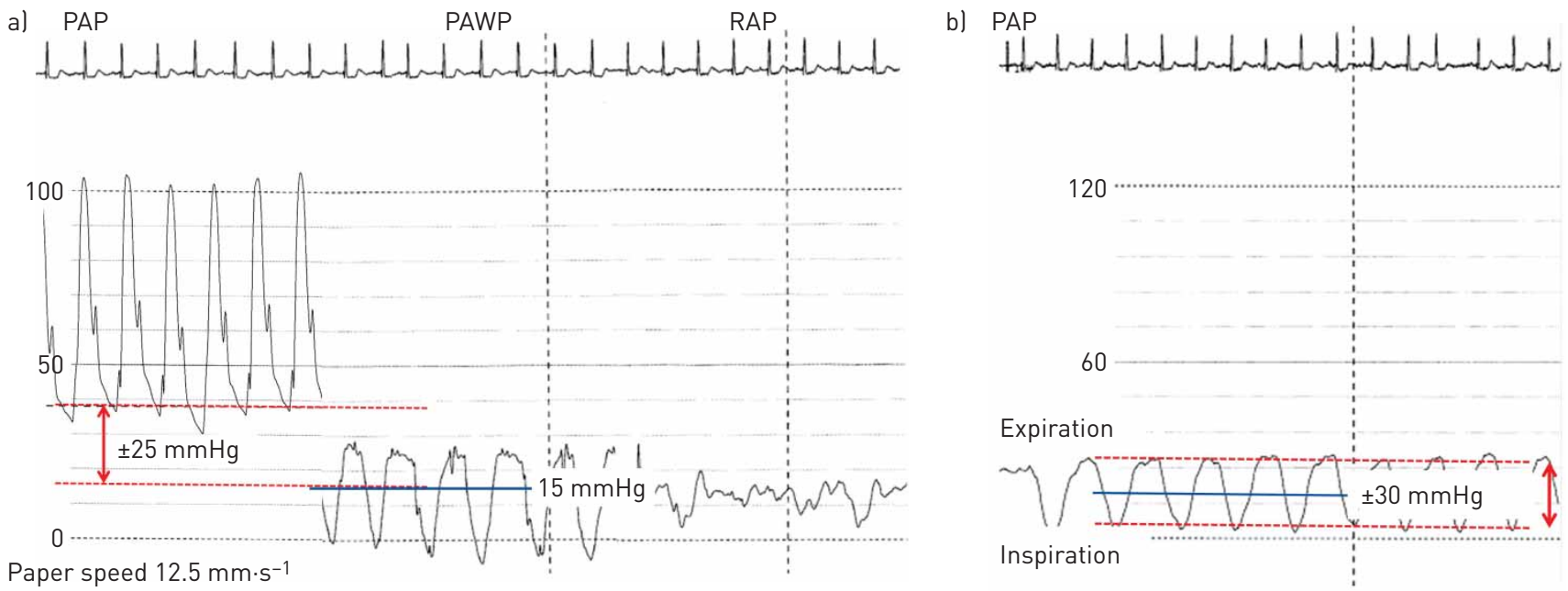

FIGURE 1 Pulmonary artery catheterisation haemodynamic tracings for case 1. a) The increase in pulmonary arterial pressure (PAP) is associated with an increase in the gradient between pulmonary artery diastolic pressure and pulmonary arterial wedge pressure (PAWP) to $25 \mathrm{mmHg}$. b) Marked respiratory variation in PAWP was observed. RAP: right atrial pressure.

15 years but had no other history of cardiac or respiratory disease and no personal or family history of thromboembolism. On examination, she had severe kyphoscoliosis, moderate-severe peripheral oedema, marked jugular venous pressure elevation and evidence of $\mathrm{PH}$. Echocardiography confirmed severe $\mathrm{PH}$ with no evidence of underlying LHD or right-left shunt on contrast bubble study (table 2). Measurement of arterial blood gases revealed chronic, compensated respiratory acidosis and marked hypoxaemia. PFTs indicated mild restrictive lung disease. Nuclear $V^{\prime} / Q^{\prime}$ lung scanning demonstrated ventilation heterogeneity due to kyphoscoliosis, with matched small, multifocal nonsegmental perfusion defects, not suggestive of chronic pulmonary emboli. An overnight polysomnogram revealed hypoventilation with rising arterial carbon dioxide tension $\left(\mathrm{PaCO}_{2}\right)$ levels and marked hypoxaemia, but no central or obstructive apnoea events. She was diagnosed with chronic respiratory failure due to kyphoscoliosis, exacerbated by nocturnal hypoventilation, and resulting in significant chronic hypoxaemia and severe pre-capillary $\mathrm{PH}$, most likely WHO Group $3 \mathrm{PH}$ due to lung disease and/or hypoxia. She was initiated on continuous noninvasive face-mask ventilation, supplemental oxygen and diuretics. After 2 weeks in hospital, she was transitioned to home and continued on nocturnal noninvasive ventilation. 6 months later, she was markedly improved clinically and had achieved near-normal daytime $\mathrm{PaCO}_{2}$ levels with resolution of hypoxaemia, as well as marked improvement in her $\mathrm{PH}$ with no evidence of RV failure.

\section{Discussion}

In this article, two cases have been selected to illustrate the complex and challenging approach to making an accurate diagnosis of the cause of $\mathrm{PH}$ and the importance of this to ensure appropriate management. The main points that will be considered are: first, multiple potential contributing illnesses and mechanisms should be considered before making a specific diagnosis of the cause of $\mathrm{PH}$; second, comorbidities can complicate the specific diagnosis of $\mathrm{PH}$; third, a multifactorial diagnostic work-up is necessary to correctly determine $\mathrm{PH}$ aetiology; and fourth, accurate diagnosis is essential to ensure appropriate management.

\section{Multiple potential causes of PH to consider when making a specific diagnosis of PH}

A number of diverse conditions can cause $\mathrm{PH}$ and this is illustrated by the clinical classification, which categorises PH into five distinct groups: WHO Group 1 (PAH), WHO Group 2 (PH-LHD), WHO Group 3 ( $\mathrm{PH}$ due to lung disease and/or hypoxia), WHO Group 4 (chronic thromboembolic pulmonary hypertension (CTEPH) and other pulmonary artery obstructions) and WHO Group 5 (PH with unclear or multifactorial mechanisms) [1, 2]. Before reaching any specific $\mathrm{PH}$ diagnosis in an individual patient, clinicians should consider all potential comorbid conditions that can lead to the development of PH $[1,2]$.

Obesity is an independent risk factor for $\mathrm{PH}[6,7]$ and may directly contribute to the development of $\mathrm{PH}[8]$. Indeed, it has been estimated that up to $5 \%$ of otherwise healthy individuals with a BMI $>30 \mathrm{~kg} \cdot \mathrm{m}^{-2}$ have moderate or severe $\mathrm{PH}$ [8]. The mechanism by which $\mathrm{PH}$ occurs in obese patients may simply be due to the fact that obese patients are at increased risk of other conditions that can cause PH, including LHD, pulmonary thromboembolism and sleep-disordered breathing [8]. Obesity is particularly common in the USA, Canada and the UK [9-11], and is expected to become more frequent in other developed [9] and 
TABLE 2 Cardiopulmonary and physiological parameters in case 2 at diagnosis and 6 months following initiation of chronic noninvasive ventilation

\begin{tabular}{|c|c|c|}
\hline Parameter & Baseline & Post-noninvasive ventilation \\
\hline \multicolumn{3}{|l|}{ Echocardiography } \\
\hline TRV $m \cdot s^{-1}$ & 4.3 & 3.3 \\
\hline RVSP mmHg & 90 & 48 \\
\hline RV size & Moderate dilation & Normal \\
\hline RV systolic function & Moderate dysfunction & Normal \\
\hline TAPSE mm & 14 & 18 \\
\hline \multicolumn{3}{|l|}{ Arterial blood gas } \\
\hline $\mathrm{pH}$ & 7.37 & 7.39 \\
\hline $\mathrm{PaCO}_{2} \mathrm{mmHg}$ & 75 & 46 \\
\hline $\mathrm{PaO}_{2}(\mathrm{mmHg})$ & 52 & 67 \\
\hline $\mathrm{HCO}_{3}^{-} \mathrm{mEq} \cdot \mathrm{L}^{-1}$ & 42 & 27 \\
\hline \multicolumn{3}{|l|}{ Pulmonary function tests } \\
\hline FEV $1 \%$ of predicted & 53 & 59 \\
\hline FVC $\%$ of predicted & 56 & 62 \\
\hline $\mathrm{FEV}_{1} / \mathrm{FVC}$ & 0.79 & 0.78 \\
\hline TLC $\%$ of predicted & 56 & 60 \\
\hline$D$ Lco $(\%)$ & 42 & 65 \\
\hline \multicolumn{3}{|l|}{ Right heart catheterisation } \\
\hline Systolic/diastolic PAP mmHg & $80 / 32$ & \\
\hline Mean PAP mmHg & 47 & \\
\hline PAWP mmHg & 12 & \\
\hline $\mathrm{DPG} \mathrm{mmHg}$ & 20 & \\
\hline $\mathrm{CO} / \mathrm{Cl} \mathrm{L} \cdot \mathrm{min}^{-1}$ & $3.8 / 2.2$ & \\
\hline PVR Wood units & 9.3 & \\
\hline RAP mmHg & 13 & \\
\hline $\mathrm{SvO}_{2} \%$ & 62 & \\
\hline
\end{tabular}

TRV: tricuspid regurgitation velocity; RVSP: right ventricular systolic pressure; RV: right ventricle/ ventricular; TAPSE: tricuspid annular plane systolic excursion; $P_{\mathrm{aCO}}$ : arterial carbon dioxide tension; $P_{\mathrm{aO}}$ : arterial oxygen pressure; $\mathrm{HCO}_{3}^{-}$: bicarbonate ion; $\mathrm{FEV}_{1}$ : forced expiratory volume in the $1 \mathrm{~s}$; $\mathrm{FVC}$ : forced vital capacity; TLC: total lung capacity; DLCO: diffusing capacity of the lung for carbon monoxide; PAP: pulmonary arterial pressure; PAWP: pulmonary artery wedge pressure; DPG: diastolic pressure gradient; $\mathrm{CO}$ : cardiac output; $\mathrm{Cl}$ : cardiac index; PVR: pulmonary vascular resistance; RAP: right atrial pressure; $\mathrm{SvO}_{2}$ mixed venous oxygen saturation.

developing [11] countries. Therefore, it is clear that in the coming years, specialist PH centres will be seeing more obese patients with $\mathrm{PH}$, like the patient illustrated in case 1.

Several types of lung diseases can lead to pre-capillary PH (WHO Group $3 \mathrm{PH}$ due to lung disease and/or hypoxia) $[1,2]$. Sleep-disordered breathing is one example, and can include obstructive sleep apnoea and nocturnal hypoventilation, which can be related to obesity [8, 12]. Sleep-disordered breathing is very common in obese patients, and screening for sleep disorders should be part of a routine work-up in obese patients with $\mathrm{PH}$ [13]. Hypoventilation both during daytime and nocturnally can also arise due to skeletal deformities such as kyphoscoliosis, as illustrated by case 2. Kyphoscoliosis is an abnormal curvature of the spine in both the anterior and lateral directions, which results in reduced chest wall compliance [14]. As such, respiratory difficulties are common in patients with significant kyphoscoliosis and chronic respiratory failure can develop [14]. $\mathrm{PH}$ is a common complication of severe kyphoscoliosis, developing as a result of hypoxaemia due to hypoventilation, and to a lesser extent, hypercapnia [14]. $\mathrm{PH}$ is also common in patients with chronic advanced lung diseases, including pulmonary fibrosis and chronic obstructive pulmonary disease, especially when both conditions are present [15]. Comorbid $\mathrm{PH}$ and chronic lung disease is associated with increased mortality and reduced functional status, as well as quality of life, highlighting the clinical need to accurately and completely diagnose all respiratory conditions in patients suspected of having $\mathrm{PH}[16,17]$.

CTEPH is an important cause of pre-capillary PH [18]. Diagnosis of this form of PH is crucial, as it can often be cured by pulmonary endarterectomy (PEA) $[1,2,18,19]$, and can also be treated with $\mathrm{PH}$-specific medications $[1,2,18]$. Patients with recurrent or multiple pulmonary emboli are at risk of CTEPH [20, 21], despite effective anticoagulation. It is recommended that specific diagnostic testing is conducted to investigate the presence of CTEPH in all patients being assessed for PH $[1,2,18,22]$. 
$\mathrm{PH}$ is a common complication of LHD, including systolic and diastolic left ventricular (LV) dysfunction and left-sided valvular disease, although the exact prevalence of PH among patients with LHD is unknown [23-26]. PH-LHD is caused by passive backward transmission of elevated filling pressures (i.e. LVEDP), and is associated with more severe symptoms, worse exercise capacity and a negative impact on clinical outcomes [23]. There are no approved therapies for PH-LHD [23]; studies have not established any benefit of PH-specific medications in patients with PH-LHD, and conversely, have reported significant adverse effects [1-3]. As a result, distinguishing PH-LHD from PAH is essential but this can be challenging [23, 27], particularly when PH is due to occult LHD (such as HFpEF as seen in case 1). One factor that contributes to the challenge of differentiating between PH-LHD and PAH is the changing demographics of PAH populations. Historically, PH-LHD patients were considered as being typically older than $\mathrm{PAH}$ patients $[1,2]$; today, $\mathrm{PAH}$ populations have a higher mean age than those in the past [28]. As the age of PAH patients increases, the likelihood of confusing PH-LHD with $\mathrm{PAH}$ also becomes higher. In part due to their increasing age, PAH patients today also have more comorbidities than in the past $[4,29]$. Indeed, recent registry data indicate that a significant proportion of PAH patients now have a comorbidity profile similar to that found in PH-LHD patients, including obesity, diabetes and atrial fibrillation. Case 1 provides an example of a patient with PH-LHD due to HFpEF with exactly such a comorbidity profile: he was morbidly obese and had been previously diagnosed with a number of chronic conditions, including type 2 diabetes mellitus and systemic hypertension. In these patients, it is important to carefully define the haemodynamic profile, using LHC in cases of uncertainty, to clearly distinguish between a diagnosis of PAH or PH-LHD.

\section{Multifactorial diagnostic assessment of patients with suspected PH}

Given the multiple potential causes of $\mathrm{PH}$ and the impact that the specific $\mathrm{PH}$ diagnosis can have on the most appropriate treatment, clinicians should perform a comprehensive multifactorial work-up to correctly determine the precise cause of $\mathrm{PH}$.

Once $\mathrm{PH}$ is suspected, echocardiography is recommended to assess heart structure and function, and to determine the probability of $\mathrm{PH}[1,2]$. In case 1 , echocardiography revealed a tricuspid valve regurgitation velocity that was above the upper limit of normal of $3.4 \mathrm{~m} \cdot \mathrm{s}^{-1}$ recommended in the European Society of Cardiology (ESC)/European Respiratory Society (ERS) guidelines [1, 2], and which indicates a high probability of $\mathrm{PH}$. Furthermore, elevated right ventricular systolic pressure is an important clue to the presence of clinically significant PH. In some cases, a structural left heart abnormality can be detected by echocardiography, raising suspicion of PH-LHD $[1,2]$. However, echocardiographic parameters suggestive of PH-LHD are not easily measurable in all patients with LHD [25]. In particular, echocardiographic findings of HFpEF are often subtle and can easily be missed [26]. This was observed in the patient in case 1; although he was ultimately diagnosed with LHD, he had no obvious echocardiographic left-sided cardiac abnormalities, and both left ventricular ejection fraction and left atrial size were normal. In patients with obesity, good quality echocardiographic images can be difficult to obtain, and this was observed for case 1. Indeed, echocardiographic images are classified as being "nondiagnostic" in up to $30 \%$ of obese patients [30, 31].

Rigorous evaluation of the potential contribution of significant lung disease is indicated during diagnostic work-up. PFTs should be conducted to provide a specific assessment of lung function and to establish important obstructive or restrictive lung disease. Indeed, for the patient in case 2, PFTs indicated moderate restrictive lung disease, which helped to reach the final diagnosis of WHO Group $3 \mathrm{PH}$ due to lung disease and/or hypoxia. Measurement of arterial blood gases established the presence of resting hypoxaemia, which was also essential in order to support this cause of $\mathrm{PH}$. Moreover, the presence of daytime or nocturnal elevated $\mathrm{PaCO}_{2}$ levels confirms hypoventilation, which is an important risk factor for WHO Group $3 \mathrm{PH}[1,2]$.

Thoracic computed tomography (CT) imaging is a valuable tool in the diagnostic approach to $\mathrm{PH}[1,2]$. $\mathrm{CT}$ findings can suggest the presence of $\mathrm{PH}$, including main or hilar pulmonary artery enlargement, $\mathrm{RV}$ enlargement, or reflux of contrast material into the inferior vena cava or hepatic veins, and can also indicate the presence of significant underlying lung disease as a cause of $\mathrm{PH}[1,2]$. In case 1 , a contrast-enhanced thoracic CT scan showed relatively small lungs (possibly due to the patient's morbid obesity) and large hilar pulmonary arteries but no evidence of parenchymal lung disease.

It is strongly recommended that in all patients with $\mathrm{PH}$, the potential contribution of chronic pulmonary emboli should be examined using a $V^{\prime} / Q^{\prime}$ lung scan $[1,2,18]$. Mismatched segmental perfusion defects indicate the possibility of CTEPH [1,2], whereas a normal or low-probability $V^{\prime} / Q^{\prime}$ scan allows CTEPH to be excluded, as in both cases presented.

Identifying the underlying cause of $\mathrm{PH}$ can be aided by performing exercise testing. Exercise testing with a 6-min walk test objectively assesses functional capacity in $\mathrm{PH}$ patients, confirming clinical severity of symptoms and exercise limitation, but does not provide any information regarding the specific cause of $\mathrm{PH}$. Moreover, 6-min walk distance values both at baseline and during follow-up can predict the risk of death in 
many types of $\mathrm{PH}[32,33]$. As such, clinical practice guidelines recommend baseline and serial assessment of exercise capacity with a 6 -min walk test $[1,2]$. More information can be provided by cardiopulmonary exercise testing (CPET), which is becoming increasingly utilised in clinical practice, and is now considered the gold standard test for evaluating the causes of dyspnoea and exercise intolerance [34, 35]. CPET can be particularly useful in the differential diagnosis of $\mathrm{PH}$ as the pattern of cardiopulmonary and gas-exchange responses can distinguish between pulmonary and cardiac limitations to exercise [35]. It must be cautioned that the proper performance and accurate interpretation of CPET in patients with PH requires significant technical and scientific expertise, as well as critical monitoring of patients in order to ensure safety.

\section{The role of heart catheterisation in PH diagnosis}

In order to definitively diagnose the cause of $\mathrm{PH}$, guidelines recommend that RHC should be performed [1, 2]. This is particularly critical for the diagnosis of pre-capillary forms of $\mathrm{PH}$, such as $\mathrm{PAH}$, that can be treated with $\mathrm{PH}$-specific medications. Despite the guideline recommendations, some patients are diagnosed with $\mathrm{PH}$ and treated with PH-specific medications without undergoing RHC as part of their assessment [36], and this can lead to inaccurate diagnoses and adverse outcomes [37]. One reason for not performing RHC may include concerns regarding the safety of the procedure [38] as cardiorespiratory, hypotensive or device-related complications can occur $[39,40]$. Despite these concerns, RHC is considered to be safe at expert centres, with low morbidity and mortality rates of $1.1 \%$ and $0.055 \%$, respectively [39]. Underuse of RHC may also be due to a lack of technical training or knowledge. The complexities of the technique, along with a lack of standardisation, can make RHC vulnerable to technical or data interpretation errors [40, 41]. To address this, guidance on optimising RHC is available and provided in table 3. Additional practical recommendations related to the specific measurement or derivation of certain haemodynamic variables have also recently been published [40]. Adoption of a standardised protocol for RHC, as well as ensuring that staff are appropriately trained, can improve the accuracy of RHC measurements and, therefore, of PH diagnosis [1, 2, 29, 40-45].

By RHC, $\mathrm{PH}$ is defined as a mean PAP $\geqslant 25 \mathrm{mmHg}$, and can be further differentiated into pre-capillary $\mathrm{PH}$ and post-capillary $\mathrm{PH}$ on the basis of the PAWP, such that PAWP $\leqslant 15 \mathrm{mmHg}$ defines pre-capillary $\mathrm{PH}$ and PAWP $>15 \mathrm{mmHg}$ indicates post-capillary $\mathrm{PH}[1,2]$. Post-capillary $\mathrm{PH}$ can exist in an isolated form or as $\mathrm{Cpc}-\mathrm{PH}[1,2]$. The haemodynamic distinction of the latter type is made based on $\mathrm{PH}$ in the presence of PAWP $>15 \mathrm{mmHg}$, as well as findings of an elevated pulmonary vascular resistance ( $>3$ Wood units) and/or an increased DPG (PAPdiastolic-PAWP $\geqslant 7 \mathrm{mmHg}$ ) $[1,2]$. In patients with post-capillary $\mathrm{PH}$, the additional pre-capillary component can be due to underlying lung disease $[1,2]$ or as a result of a pulmonary vascular remodelling process in the setting of chronic LHD, as in mitral stenosis, LV systolic

TABLE 3 Potential sources of error when performing right heart catheterisation and guidance on how to minimise the impact of these errors

\section{Potential error class}

\section{Potential error}

Risk

Solution

Measurement

Data interpretation

Technical

\section{Catheter balloon} over-inflation

Catheter balloon under-inflation Use of end-expiratory PAWP readings

Analysing single cycle

Variation in the location of the pressure transducer

Failure to review traces

Incorrectly maintained or calibrated equipment Inadequate flushing of the catheter
False high or low PAWP readings [41]

Pulmonary arterial rupture [41]

False elevation of PAWP readings [41]

Misdiagnosis of patients with a pre-capillary phenotype $[43,44]$

Potential data inaccuracy [44]

Nonuniformity of the pressure transducer setting and zero levelling $[29,45]$

Data inaccuracy (measurement artefacts) [41]

Errors in data acquisition [41]

Dampened waveforms [41]
Half inflation of the balloon (diameter $0.9 \mathrm{~cm}, 0.75 \mathrm{~mL}$ air) [42] Avoid repeated inflations and deflations of the balloon [29]

Half inflation of the balloon (diameter $\sim 0.9 \mathrm{~cm}, 0.75 \mathrm{~mL}$ air) [42] Average PAWP readings across respiratory cycles [44]

Mean values of multiple respiratory cycles should be used [44]

Standardised location of pressure transducer according to guideline recommendations $[1,2,40,44]$ or adoption of micromanometer-tipped catheters [44]

Each trace should be scrutinised to ensure that it is not affected by artefacts [41]

Equipment maintained to a high standard and regular calibration [41] Adequate flushing [41]

PAWP: pulmonary arterial wedge pressure. 
dysfunction or HFpEF [23-25]. In case 1, RHC revealed the presence of significant PH (table 1), with elevated PAP (normal mean PAP $\leqslant 20 \mathrm{mmHg}[1,2,29]$ ) and increased pulmonary vascular resistance (normal $\leqslant 1.5$ Wood units [46, 47]). In addition, a PAWP of $16 \mathrm{mmHg}$ was measured (figure 1a), which is close to the cut-off point of $\leqslant 15 \mathrm{mmHg}$ described above $[1,2]$. However, because of the obesity, there were marked respiratory swings in the RHC trace which can affect the accuracy of the PAWP reading (figure 1b). If the PAWP value is unreliable or if there are significant risk factors for LHD (both of which occurred in case 1), the current ESC/ERS guidelines state that LHC should be considered to directly measure LVEDP [1, 2]. LVEDP is the gold standard measurement for distinguishing between pre- and post-capillary $\mathrm{PH}$. In contrast, PAWP is only an estimate and can be affected by many factors, including respiratory swings [40]. The LHC results in case 1 revealed an elevated DPG. The degree of elevation of mean PAP in this patient could not be explained entirely by the PAWP and LVEDP values, suggesting the presence of a pre-capillary component to this patient's $\mathrm{PH}$. Therefore, the most appropriate diagnosis for the patient in case 1 was WHO Group 2 PH-LHD with Cpc-PH, complicated by morbid obesity. The post-capillary component was a result of HFpEF. The pre-capillary component was likely the result of pulmonary vascular remodelling in the setting of HFpEF.

Invasive measurement of cardiopulmonary haemodynamics during exercise rather than at rest as described above may provide additional diagnostic value. Patients with HFpEF in the early stages of the disease may have normal haemodynamic values at rest or may have haemodynamic values suggestive of $\mathrm{PH}$ (defined as high resting PAP, but with normal PAWP and/or LVEDP [ $\leqslant 15 \mathrm{mmHg}]$ ). However, when the cardiovascular system is stressed and cardiac output increases during exercise, pathological changes in systolic PAP, mean PAP and PAWP may occur [48]. Haemodynamic testing during exercise can therefore "unmask" diastolic dysfunction, aiding the early diagnosis of HFpEF [49]. Exercise haemodynamics are especially useful in obese patients [49] who, as seen in case 1, often present with unreliable PAWP values. As invasive exercise testing is not universally available, fluid loading can be used as an alternative method of stressing the cardiovascular system, although it may be less sensitive than exercise testing [48]. Currently, the methodology of haemodynamic exercise testing in the diagnosis of $\mathrm{PH}$ is not standardised and guidelines do not recommend it as a routine diagnostic test [1, 2, 49]. A recent publication has suggested a diagnostic algorithm for interpreting the haemodynamic and ventilatory data that CPET with invasive haemodynamic assessment provides [50], which may help to standardise this technique in the future.

To identify the correct cause of $\mathrm{PH}$, particularly in the setting of patients who have multiple comorbidities, a comprehensive, multidimensional diagnostic methodology is required, as illustrated by the two cases presented. Due to the increasing complexity of diagnosing $\mathrm{PH}$, it is recommended that patients presenting with suspected $\mathrm{PH}$ are referred to a specialist $\mathrm{PH}$ centre [15]. Furthermore, clinicians working outside of these centres are encouraged to discuss complex cases with $\mathrm{PH}$ specialists to aid a timely and accurate diagnosis.

The implications of PH diagnosis on patient management

Failure to reach a correct diagnosis of the cause of $\mathrm{PH}$ in an individual patient can result in patients receiving inappropriate treatments for their specific type of $\mathrm{PH}$. $\mathrm{PH}$-targeted medications are approved and effective in patients with PAH $[1,2]$, but are not indicated for many of the other forms of PH.

For patients with PH-LHD, PH-specific therapies are not recommended or approved [1-3] as they have demonstrated a lack of efficacy in patients with HFpEF and those with $\mathrm{PH}$ due to systolic LV dysfunction [51-54]. Moreover, in patients with heart failure and a reduced ejection fraction, $\mathrm{PH}$-specific therapies have increased the occurrence of adverse events [55] or hastened disease progression as a consequence of fluid retention [56]. Similarly, the use of drugs approved for $\mathrm{PH}$ is not recommended in patients with $\mathrm{PH}$ due to lung diseases $[1,2]$. This highlights the critical importance of differentiating PAH from WHO Group $2 \mathrm{PH}-\mathrm{LHD}$ or WHO Group $3 \mathrm{PH}$ due to lung disease and/or hypoxia prior to initiating $\mathrm{PH}$-targeted medical therapy. The optimal management of CTEPH patients is different again to that of other forms of $\mathrm{PH}$, with surgical PEA being the treatment of choice in eligible patients $[1,2]$. Medical treatment of CTEPH patients with $\mathrm{PH}$-targeted therapy may be justified for inoperable patients or for patients with persistent or recurrent $\mathrm{PH}$ following PEA $[1,2]$.

For the management of PH in WHO Groups 2 and 3, treatment of the underlying cause/comorbidity is essential and is recommended in the ESC/ERS guidelines for $\mathrm{PH}[1,2]$. Treatment of comorbidities in $\mathrm{PH}$ patients is important as it has been demonstrated that certain comorbidities are associated with worse outcomes in patients with PH. For example, long-term survival appears worse in $\mathrm{PH}$ patients with diabetes in comparison to $\mathrm{PH}$ patients without diabetes [57]. Furthermore, a recent meta-analysis has demonstrated that bariatric surgery leads to short-term clinical improvements in PH in obese patients [58]. In alignment with this, in case 1 , the patient underwent a sleeve gastric band procedure, which led to significant improvements in his condition. 2 years after surgery, the patient's BMI had reduced to $36.6 \mathrm{~kg} \cdot \mathrm{m}^{-2}$. Follow-up echocardiography indicated that he was largely cured of his PH. There was a qualitative improvement in RV 
size/left ventricle compression and right atrium size. Treatment of underlying chronic hypoventilation due to kyphoscoliosis using noninvasive ventilation also markedly improved $\mathrm{PH}$ in case 2 . This is in line with previous data on the treatment of chronic hypoventilation syndromes using ventilation therapy. In a retrospective analysis that assessed patients with daytime $\mathrm{PH}$ due to hypoventilation, noninvasive ventilation was found to significantly improve haemodynamics and exercise capacity [59].

In addition to highlighting the benefits of treating comorbidities, case 1 also illustrates how failure to reach a definitive diagnosis can have significant implications for the management of $\mathrm{PH}$ patients. Undiagnosed conditions can result in patients being rejected for potentially beneficial procedures. The patient in case 1 had previously been refused bariatric surgery, as there was a perceived risk of operating in the setting of an unidentified cardiopulmonary condition. Following successful diagnosis of $\mathrm{PH}$, and discussions between specialist surgical and cardiology staff, the original decision not to operate was overturned as surgeons felt that the $\mathrm{PH}$ was reversible.

\section{Conclusion}

As illustrated in this review, the accurate diagnosis of the underlying cause of $\mathrm{PH}$ is associated with a number of challenges. There are multiple cardiac and pulmonary conditions that can contribute to the development of $\mathrm{PH}$, and the frequency and variety of comorbid conditions complicates the diagnostic approach to a $\mathrm{PH}$ patient. It is, however, essential that the type of $\mathrm{PH}$ is correctly identified, in order to ensure that patients are optimally managed.

\section{Acknowledgements}

We would like to thank James Glasper (nspm Ltd, Meggen, Switzerland) for medical writing assistance, funded by Actelion Pharmaceuticals Ltd (Allschwil, Switzerland).

\section{References}

1 Galiè N, Humbert M, Vachiery JL, et al. 2015 ESC/ERS Guidelines for the diagnosis and treatment of pulmonary hypertension. Eur Heart J 2016; 37: 67-119.

2 Galiè N, Humbert M, Vachiery JL, et al. 2015 ESC/ERS Guidelines for the diagnosis and treatment of pulmonary hypertension. Eur Respir J 2015; 46: 903-975.

3 Farber HW, Gibbs S. Under pressure: pulmonary hypertension associated with left heart disease. Eur Respir Rev 2015; 24: 665-673.

4 Ling Y, Johnson MK, Kiely DG, et al. Changing demographics, epidemiology, and survival of incident pulmonary arterial hypertension: results from the pulmonary hypertension registry of the United Kingdom and Ireland. Am J Respir Crit Care Med 2012; 186: 790-796.

5 Hoeper MM, Simon RGJ. The changing landscape of pulmonary arterial hypertension and implications for patient care. Eur Respir Rev 2014; 23: 450-457.

6 Leung CC, Moondra V, Catherwood E, et al. Prevalence and risk factors of pulmonary hypertension in patients with elevated pulmonary venous pressure and preserved ejection fraction. Am J Cardiol 2010; 106: 284-286.

7 Choudhary G, Jankowich M, Wu WC. Prevalence and clinical characteristics associated with pulmonary hypertension in African-Americans. PLoS One 2013; 8: e84264.

8 Friedman SE, Andrus BW. Obesity and pulmonary hypertension: a review of pathophysiologic mechanisms. J Obes 2012; 2012: 505274.

9 Twells LK, Gregory DM, Reddigan J, et al. Current and predicted prevalence of obesity in Canada: a trend analysis. CMAJ Open 2014; 2: E18-E26.

10 Ogden CL, Carroll MD, Fryar CD, et al. Prevalence of obesity among adults and youth: United States, 2011-2014. NCHS Data Brief 2015; 1-8.

$11 \mathrm{Ng} \mathrm{M}$, Fleming T, Robinson $\mathrm{M}$, et al. Global, regional, and national prevalence of overweight and obesity in children and adults during 1980-2013: a systematic analysis for the Global Burden of Disease Study 2013. Lancet 2014; 384: 766-781.

12 Arias MA, Garcia-Rio F, Alonso-Fernandez A, et al. Pulmonary hypertension in obstructive sleep apnoea: effects of continuous positive airway pressure: a randomized, controlled cross-over study. Eur Heart J 2006; 27: 1106-1113.

13 Kholdani C, Fares WH, Mohsenin V. Pulmonary hypertension in obstructive sleep apnea: is it clinically significant? A critical analysis of the association and pathophysiology. Pulm Circ 2015; 5: 220-227.

14 Weinberger SE, Cockrill BA, Mandel J. Principles of Pulmonary Medicine. 5th Edn. Saunders Elsevier, 2008.

15 Seeger W, Adir Y, Barbera JA. Pulmonary hypertension in chronic lung diseases. J Am Coll Cardiol 2013; 62: D109-D116.

16 Hayes D Jr, Black SM, Tobias JD, et al. Influence of pulmonary hypertension on survival in advanced lung disease. Lung 2015; 193: 213-221.

17 Cuttica MJ. Pulmonary hypertension associated with lung diseases and hypoxemia. Heart Fail Rev 2016; 21: 299-308.

18 Mehta S, Helmersen D, Provencher S, et al. Diagnostic evaluation and management of chronic thromboembolic pulmonary hypertension: a clinical practice guideline. Can Respir J 2010; 17: 301-334.

19 McNeil K, Dunning J. Chronic thromboembolic pulmonary hypertension (CTEPH). Heart 2007; 93: 1152-1158.

20 Lang I. Chronic thromboembolic pulmonary hypertension: a distinct disease entity. Eur Respir Rev 2015; 24: $246-252$.

21 Yang S, Yang Y, Zhai Z, et al. Incidence and risk factors of chronic thromboembolic pulmonary hypertension in patients after acute pulmonary embolism. J Thorac Dis 2015; 7: 1927-1938.

22 Lang IM, Madani M. Update on chronic thromboembolic pulmonary hypertension. Circulation 2014; 130: 508-518.

23 Vachiéry JL, Adir Y, Barbera JA. Pulmonary hypertension due to left heart diseases. J Am Coll Cardiol 2013; 62: D100-D108. 
31 Mulvagh SL, DeMaria AN, Feinstein SB, et al. Contrast echocardiography: current and future applications. $J$ Am Soc Echocardiogr 2000; 13: 331-342.

32 Humbert M, Sitbon O, Chaouat A, et al. Survival in patients with idiopathic, familial, and anorexigen-associated pulmonary arterial hypertension in the modern management era. Circulation 2010; 122: 156-163.

33 Farber HW, Miller DP, McGoon MD, et al. Predicting outcomes in pulmonary arterial hypertension based on the 6-minute walk distance. J Heart Lung Transplant 2015; 34: 362-368.

34 Ferrazza AM, Martolini D, Valli G, et al. Cardiopulmonary exercise testing in the functional and prognostic evaluation of patients with pulmonary diseases. Respiration 2009; 77: 3-17.

35 Palange P, Ward SA, Carlsen KH, et al. Recommendations on the use of exercise testing in clinical practice. Eur Respir J 2007; 29: 185-209.

36 McLaughlin VV, Langer A, Tan M, et al. Contemporary trends in the diagnosis and management of pulmonary arterial hypertension: an initiative to close the care gap. Chest 2013; 143: 324-332.

37 Deano RC, Glassner-Kolmin C, Rubenfire M, et al. Referral of patients with pulmonary hypertension diagnoses to tertiary pulmonary hypertension centers: the multicenter RePHerral study. JAMA Intern Med 2013; 173: 887-893.

38 Zuckerman WA, Turner ME, Kerstein J, et al. Safety of cardiac catheterization at a center specializing in the care of patients with pulmonary arterial hypertension. Pulm Circ 2013; 3: 831-839.

39 Hoeper MM, Lee SH, Voswinckel R, et al. Complications of right heart catheterization procedures in patients with pulmonary hypertension in experienced centers. J Am Coll Cardiol 2006; 48: 2546-2552.

40 Rosenkranz S, Preston IR. Right heart catheterisation: best practice and pitfalls in pulmonary hypertension. Eur Respir Rev 2015; 24: 642-652.

41 Mathier M. The nuts and bolts of interpreting hemodynamics in pulmonary hypertension associated with diastolic heart failure. Adv Pulm Hypertens J 2011; 10: 33-40.

42 Tonelli AR, Mubarak KK, Li N, et al. Effect of balloon inflation volume on pulmonary artery occlusion pressure in patients with and without pulmonary hypertension. Chest 2011; 139: 115-121.

43 LeVarge BL, Pomerantsev E, Channick RN. Reliance on end-expiratory wedge pressure misclassifies pulmonary hypertension. Eur Respir J 2014; 44: 425-434.

44 Kovacs G, Avian A, Pienn M, et al. Reading pulmonary vascular pressure tracings. How to handle the problems of zero leveling and respiratory swings. Am J Respir Crit Care Med 2014; 190: 252-257.

45 Kovacs G, Avian A, Olschewski A, et al. Zero reference level for right heart catheterisation. Eur Respir J 2013; 42: $1586-1594$.

46 Kovacs G, Berghold A, Scheidl S, et al. Pulmonary arterial pressure during rest and exercise in healthy subjects: a systematic review. Eur Respir J 2009; 34: 888-894.

47 Baim DS, ed. Grossman's Cardiac Catheterization, Angiography, and Intervention. 7th Edn. Philadelphia, Lippincott Williams \& Wilkins, 2006.

48 Andersen MJ, Olson TP, Melenovsky V, et al. Differential hemodynamic effects of exercise and volume expansion in people with and without heart failure. Circ Heart Fail 2015; 8: 41-48.

49 Maor E, Grossman Y, Balmor RG, et al. Exercise haemodynamics may unmask the diagnosis of diastolic dysfunction among patients with pulmonary hypertension. Eur J Heart Fail 2015; 17: 151-158.

50 Berry NC, Manyoo A, Oldham WM, et al. Protocol for exercise hemodynamic assessment: Performing an invasive cardiopulmonary exercise test in clinical practice. Pulm Circ 2015; 5: 610-618.

51 Hoendermis ES, Liu LC, Hummel YM, et al. Effects of sildenafil on invasive haemodynamics and exercise capacity in heart failure patients with preserved ejection fraction and pulmonary hypertension: a randomized controlled trial. Eur Heart J 2015; 36: 2565-2573.

52 Borlaug BA, Lewis GD, McNulty SE, et al. Effects of sildenafil on ventricular and vascular function in heart failure with preserved ejection fraction. Circ Heart Fail 2015; 8: 533-541.

53 Redfield MM, Chen HH, Borlaug BA, et al. Effect of phosphodiesterase-5 inhibition on exercise capacity and clinical status in heart failure with preserved ejection fraction: a randomized clinical trial. JAMA 2013; 309: $1268-1277$.

54 Bonderman D, Ghio S, Felix SB, et al. Riociguat for patients with pulmonary hypertension caused by systolic left ventricular dysfunction: a phase IIb double-blind, randomized, placebo-controlled, dose-ranging hemodynamic study. Circulation 2013; 128: 502-511.

55 Packer M, McMurray J, Massie BM, et al. Clinical effects of endothelin receptor antagonism with bosentan in patients with severe chronic heart failure: results of a pilot study. J Card Fail 2005; 11: 12-20.

56 Kalra PR, Moon JC, Coats AJ. Do results of the ENABLE (Endothelin Antagonist Bosentan for Lowering Cardiac Events in Heart Failure) study spell the end for non-selective endothelin antagonism in heart failure? Int J Cardiol 2002; 85: 195-197.

57 Abernethy $\mathrm{AD}$, Stackhouse $\mathrm{K}$, Hart S, et al. Impact of diabetes in patients with pulmonary hypertension. Pulm Circ 2015; 5: 117-123.

58 Sheu EG, Channick R, Gee DW. Improvement in severe pulmonary hypertension in obese patients after laparoscopic gastric bypass or sleeve gastrectomy. Surg Endosc 2016; 30: 633-637.

59 Held M, Walthelm J, Baron S, et al. Functional impact of pulmonary hypertension due to hypoventilation and changes under noninvasive ventilation. Eur Respir J 2014; 43: 156-165. 